\title{
Morcegos em área de Floresta Montana, Visconde de Mauá, Resende, Rio de Janeiro
}

\author{
Júlia Lins Luz $z^{1,4}$, Luciana de Moraes Costa ${ }^{1,2}$, Tássia Jordão-Nogueira ${ }^{3}$, \\ Carlos Eduardo Lustosa Esbérard ${ }^{1} \&$ Helena de Godoy Bergallo ${ }^{3}$ \\ ${ }^{1}$ Laboratório de Diversidade de Morcegos, Instituto de Biologia, Universidade Federal Rural do Rio de \\ Janeiro - UFRRJ, Km 47 da antiga estrada Rio-São Paulo, CP 74507, CEP 23890-000, Seropédica, RJ, Brasil \\ ${ }^{2}$ Programa de Pós-graduação em Ecologia e Evolução, Departamento de Ecologia, \\ Instituto de Biologia, Universidade do Estado do Rio de Janeiro - UERJ, \\ Rua São Francisco Xavier, 524, CEP 20559-900, Rio de Janeiro, RJ, Brasil \\ ${ }^{3}$ Departamento de Ecologia, Instituto de Biologia, Universidade do Estado do Rio de Janeiro - UERJ, \\ Rua São Francisco Xavier, 524, CEP 20559-900, Rio de Janeiro, RJ, Brasil \\ ${ }^{4}$ Autor para correspondência: Julia Lins Luz,e-mail: julialinsluz@yahoo.com.br
}

LUZ, J.L., COSTA, L.M., JORDÃO-NOGUEIRA, T., ESBÉRARD, C.E.L. \& BERGALLO, H.G. Bats from a Montane Forest, Visconde de Mauá, Resende, Rio de Janeiro. Biota Neotrop. 13(2): http://www.biotaneotropica. org.br/v13n2/en/abstract?inventory+bn02513022013

Abstract: The goal of this study is to provide a list of bats from Visconde de Mauá, municipality of Resende, Rio de Janeiro State, located 1,300 $\mathrm{m}$ above sea level. Fieldwork was carried out during seven nights in November 2005, with captures along flyways, feeding sites, and roosts. A total of 13 species was recorded (10 Phyllostomidae, two Vespertilionidae, and one Molossidae). Seven species are frugivores, two nectarivores, one hematophagous, and three insectivores. Sturnira lilium was the dominant species, with $79.6 \%$ of the captures.

Keywords: high-altitude, Atlantic Rainforest, bat inventory, Chiroptera, Mammalia.

LUZ, J.L., COSTA, L.M., JORDÃO-NOGUEIRA, T., ESBÉRARD, C.E.L. \& BERGALLO, H.G. Morcegos em área de Floresta Montana, Visconde de Mauá, Resende, Rio de Janeiro. Biota Neotrop. 13(2): http://www. biotaneotropica.org.br/v13n2/pt/abstract?inventory+bn02513022013

Resumo: O objetivo deste trabalho é apresentar uma lista de morcegos da região de Visconde de Mauá, município de Resende, Estado do Rio de Janeiro, em área de Floresta Ombrófila Densa Montana, localizada acima de $1.300 \mathrm{~m}$ de altitude. Foram realizadas sete noites de captura, em novembro de 2005, com amostragens em áreas de voo e forrageio e em abrigos. O total de 13 espécies foi obtido (10 Phyllostomidae, duas Vespertilionidae e uma Molossidae). Dessas, sete espécies são frugívoras, duas nectarívoras, uma hematófaga e três insetívoras. A espécie dominante foi Sturnira lilium com $79,6 \%$ das capturas.

Palavras-chave: altitude elevada, Mata Atlântica, inventário de morcegos, Chiroptera, Mammalia. 


\section{Introdução}

Diversos levantamentos de mamíferos têm sido realizados no sudeste do Brasil e mais especificamente no Estado do Rio de Janeiro (e.g., Olifiers et al. 2007, Modesto et al. 2008, Luz et al. 2011, Delciellos et al. 2012). Porém, ainda existem áreas com pouca ou nenhuma amostragem para os diferentes grupos taxonômicos (Bergallo et al. 2009). As faixas de altitudes mais elevadas estão entre essas áreas pouco amostradas (e.g., Geise et al. 2004, Olifiers et al. 2007), sendo estas de extrema importância, uma vez que os gradientes altitudinais podem influenciar a ocorrência e a abundância das espécies de mamíferos, incluindo os morcegos (Patterson et al. 1998, Geise et al. 2004, McCain 2005, 2007).

No Estado do Rio de Janeiro dois parques nacionais, que apresentam cotas altitudinais elevadas e Floresta Ombrófila Densa Montana, foram estudados quanto à composição da fauna de mamíferos em diferentes altitudes. Geise et al. (2004) registraram 69 espécies de mamíferos terrestres pertencentes a sete ordens e 20 famílias para o Parque Nacional do Itatiaia e seu entorno e Olifiers et al. (2007) registraram 11 espécies de marsupiais e 17 espécies de roedores no Parque Nacional da Serra dos Órgãos. No entanto, esses levantamentos não incluíram a ordem Chiroptera, havendo poucas áreas em altitudes mais elevadas amostradas para esse grupo (Dias et al. 2010).

Alguns levantamentos da fauna de quirópteros foram realizados nas últimas décadas nos remanescentes de Floresta Ombrófila Densa Montana localizados nas Serras do Mar e da Mantiqueira, em altitudes compreendidas entre os 500 e os 1.500 m (Esbérard et al. 1996, Dias \& Peracchi 2008, Modesto et al. 2008, Moratelli \& Peracchi 2007, Martins 2011, Delciellos et al. 2012). No entanto, no município de Resende há, até o momento, o registro de apenas duas espécies de morcegos: Diphylla ecaudata Spix, 1823 e Molossus rufus E. Geoffroy, 1805 (Esbérard 2004, Dias et al. 2010).

O objetivo deste trabalho é apresentar uma lista de morcegos da região de Visconde de Mauá, município de Resende, Estado do Rio de Janeiro, em área de Floresta Ombrófila Densa Montana, localizada acima de $1.300 \mathrm{~m}$ de altitude.

\section{Material e Métodos}

A região de Visconde de Mauá, município de Resende, localizase em terras altas da Serra da Mantiqueira, que apresentam altitude média de $1.200 \mathrm{~m}$, e dista cerca de $185 \mathrm{~km}$ da cidade do Rio de Janeiro (Bauer \& Pacheco 2000). Esta região situa-se no entorno do Parque Nacional do Itatiaia e está inserida na Área de Proteção Ambiental (APA) da Serra da Mantiqueira, criada em 1985. AAPA apresenta uma área total de 421.804 ha e ocupa parte de 27 municípios dos estados de São Paulo, Rio de Janeiro e Minas Gerais (Instituto... 2012).

O relevo é bastante acidentado e a altitude da área amostrada varia de 1.350 a 1.750 metros (Vrcibradic et al. 2010). As coletas foram realizadas em uma propriedade particular, a Fazenda Marimbondo $\left(22^{\circ} 22^{\prime} \mathrm{S}\right.$ e $44^{\circ} 35^{\prime} \mathrm{W}$ - Figura 1$)$. A localidade encontra-se inserida na Formação de Floresta Ombrófila Densa Montana caracterizada por apresentar um extrato dominante tendo o dossel altura até $25 \mathrm{~m}$ (Ururahy et al. 1983).

A amostragem de morcegos ocorreu entre os dias 05 e 11 de novembro de 2005. Foram utilizadas redes de neblina medindo de sete a $12 \mathrm{~m}$ de comprimento armadas em diferentes locais a cada noite, incluindo trilhas já existentes, coleções de água e proximidades de refúgios. Foram realizadas sete noites de captura e o número de redes por noite variou de cinco a nove. As redes foram abertas minutos antes do crepúsculo e permaneceram abertas por um período que variou

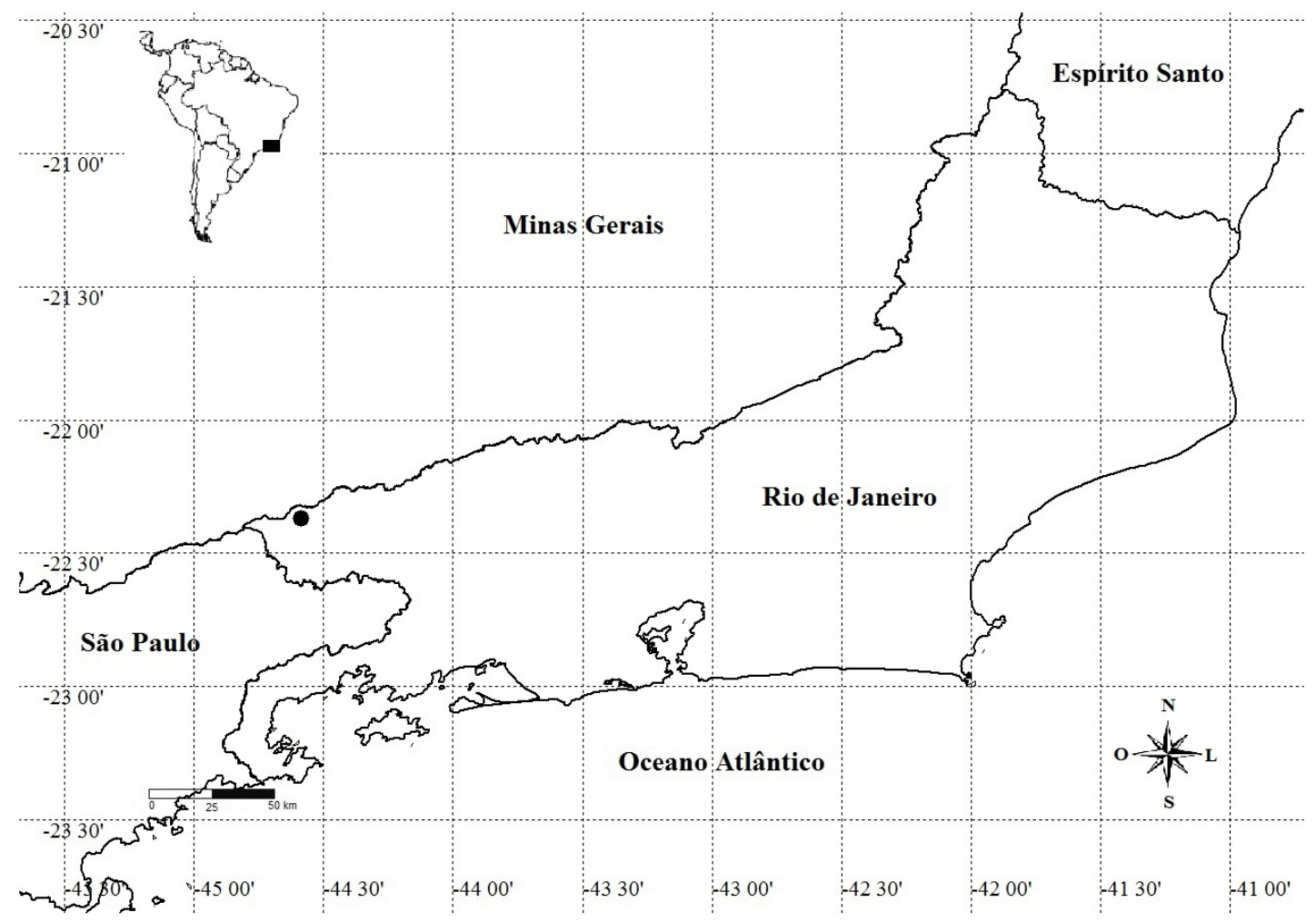

Figura 1. Mapa do Estado do Rio de Janeiro, com a localidade de Visconde de Mauá indicada pelo ponto preto.

Figure 1. Map of Rio de Janeiro State, with the location of Visconde de Mauá indicated by the black dot. 
de quatro a 12,5 horas. O esforço foi calculado segundo Straube e Bianconi (2002) e totalizou $10.135 \mathrm{~m}^{2}$.h. Foi realizada ainda a captura manual de morcegos, durante o dia, entre as telhas de um dos telhados da propriedade, apontado como refúgio pelo proprietário.

Os morcegos capturados foram identificados, mensurados (massa e antebraço), averiguados quanto ao sexo e soltos no próprio local de captura após receberem furos no dactilopatágio ("punch-marking") (Bonaccorso \& Smythe 1972). A nomenclatura das espécies seguiu Simmons (2005). Exemplares testemunhos foram depositados na coleção de referência do Laboratório de Diversidade de Morcegos (Processo 1755/89 - IBAMA/SUPES/RJ) da Universidade Federal Rural do Rio de Janeiro (Apêndice 1).

As espécies foram classificadas em guildas tróficas (carnívoros, catadores, frugívoros, hematófagos, nectarívoros, insetívoros ou onívoros) segundo Bonaccorso (1979) e foi feita uma curva aleatorizada de acumulação de espécies, com 1.000 iterações no programa Past 2.0, utilizando apenas as capturas em redes de neblina. A riqueza esperada de morcegos foi estimada através do estimador Chao 2 (Chao 1984), que leva em consideração a frequência das espécies.

\section{Resultados}

Foram analisados 204 indivíduos dos quais 10 foram recapturados, sendo nove recapturas de Sturnira lilium (E. Geoffroy, 1810) e uma de Vampyressa pusilla (Wagner, 1843). O total de 13 espécies foi obtido, sendo 10 da família Phyllostomidae, duas da família Vespertilionidae e uma da família Molossidae. Dessas, sete espécies são frugívoras, duas nectarívoras, uma hematófaga e três insetívoras (Tabela 1).

A espécie dominante foi $S$. lilium com $79,6 \%$ das capturas realizadas com redes de neblina. As outras espécies, registradas através das redes de neblina, apresentaram menos de 10 capturas sendo que Artibeus lituratus (Olfers, 1818), Carollia perspicillata (Linnaeus, 1758), Myotis riparius Handley, 1960 e Sturnira tildae de la Torre, 1959 foram representadas por apenas uma captura cada (Tabela 1). A curva de acúmulo de espécies não apresentou estabilização (Figura 2) e a riqueza estimada pelo o índice de Chao 2 foi de 21,17 .
No refúgio foram capturadas duas espécies que não haviam sido registradas em redes: Eptesicus furinalis (d'Orbigny \& Gervais, 1847) e Molossus molossus (Pallas, 1766).

\section{Discussão}

Apesar do pequeno esforço de captura, foram registradas 11,5\% das espécies de morcegos reconhecidas para a Mata Atlântica (Paglia et al. 2012) e cerca de $31 \%$ das reconhecidas para região (Dias et al. 2010). A curva de acúmulo de espécies sugere que, com o aumento do esforço de coleta no local, mais táxons serão registrados. Segundo o índice de Chao 2 a amostragem representa apenas $61,4 \%$ da riqueza esperada. O número de espécies esperado apresenta um alto valor em relação ao observado devido ao curto tempo de inventário, que não foi capaz de descrever, por completo, a fauna de morcegos local.

A captura em refúgio foi responsável por acrescentar duas espécies, da guilda de insetívoros, ao inventário de morcegos, demonstrando a importância de se utilizar mais de um método de captura. Essa guilda, em geral, é sub-amostrada em levantamentos realizados com redes de neblina (Voss \& Emmons 1996, Simmons \& Voss 1998, Esbérard 2004, Esbérard \& Bergallo 2005), pois essas espécies voam acima da copa das árvores e apresentam um sonar mais apurado (Simmons \& Voss 1998, Schnitzler \& Kalko 2001). No entanto, o uso de telhados de residências e outras construções humanas como abrigo por algumas espécies desta guilda é relatado com frequência (Esbérard et al. 1999, Luz et al. 2009).

A riqueza obtida está de acordo com aquela encontrada em inventários rápidos situados na mesma altitude e formação vegetal (Modesto et al. 2008, Delciellos et al. 2012) e a predominância de S. lilium, assim como da guilda de frugívoros, nessas áreas também já havia sido observada (Moratelli \& Peracchi 2007, Modesto et al. 2008, Nobre et al. 2009, Martins 2011). No entanto, no presente estudo, a espécie mais abundante representou cerca de dois terços das capturas, apresentando uma abundância relativa acima do encontrado nos outros estudos.

Tabela 1. Lista de espécies, número de capturas e guilda trófica de morcegos da Fazenda Marimbondo, Visconde de Mauá, município de Resende, RJ, capturados em novembro de 2005. As recapturas estão indicadas entre parênteses.

Table 1. Species list, number of captures and trophic guild of bats from Fazenda Marimbondo, Visconde de Mauá, Resende, RJ, captured in November 2005. The recaptures are indicated in parentheses.

\begin{tabular}{|c|c|c|c|}
\hline Espécies & Capturas em rede & Capturas em refúgio & Guilda descrita \\
\hline \multicolumn{4}{|l|}{ Família Phyllostomidae } \\
\hline Anoura caudifer (E. Geoffroy, 1818) & 7 & - & Nectarívoro \\
\hline Anoura geoffroyi Gray, 1838 & 8 & - & Nectarívoro \\
\hline Artibeus fimbriatus Gray, 1838 & 3 & - & Frugívoro \\
\hline Artibeus lituratus (Olfers, 1818) & 1 & - & Frugívoro \\
\hline Carollia perspicillata (Linnaeus, 1758) & 1 & - & Frugívoro \\
\hline Desmodus rotundus (E. Geoffroy, 1810) & 4 & - & Hematófago \\
\hline Platyrrhinus recifinus (Thomas, 1901) & 4 & - & Frugívoro \\
\hline Sturnira lilium (E. Geoffroy, 1810) & $133(9)$ & - & Frugívoro \\
\hline Sturnira tildae de la Torre, 1959 & 1 & - & Frugívoro \\
\hline Vampyressa pusilla (Wagner, 1843) & $4(1)$ & - & Frugívoro \\
\hline \multicolumn{4}{|l|}{ Família Molossidae } \\
\hline Molossus molossus (Pallas, 1766) & - & 4 & Insetívoro \\
\hline \multicolumn{4}{|l|}{ Família Vespertilionidae } \\
\hline Eptesicus furinalis (d’Orbigny \& Gervais, 1847) & - & 33 & Insetívoro \\
\hline Myotis riparius Handley, 1960 & 1 & - & Insetívoro \\
\hline
\end{tabular}




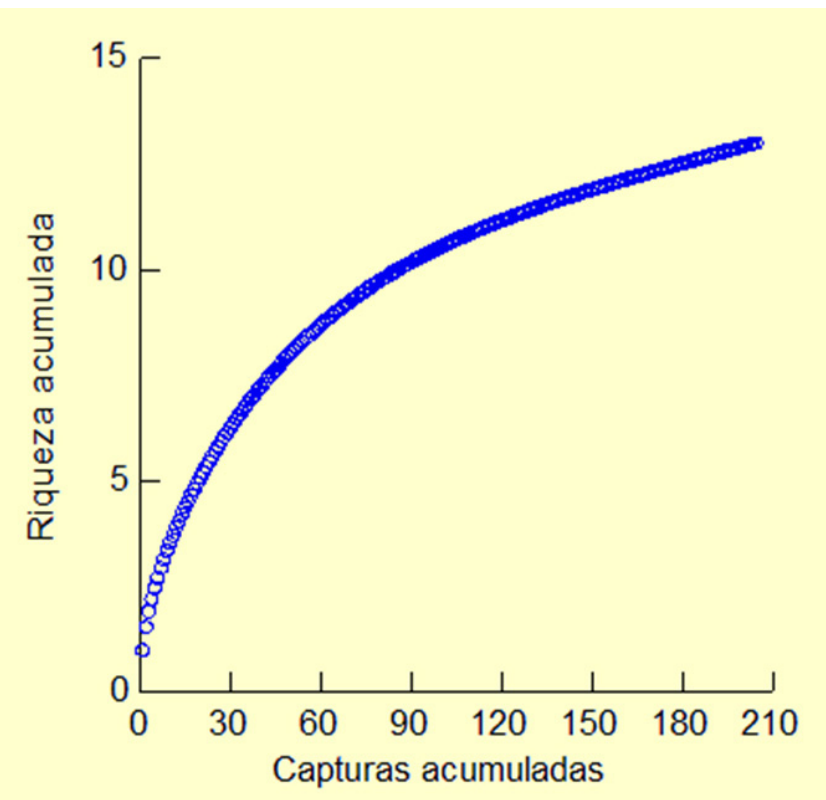

Figura 2. Curva aleatorizada (com desvio padrão) de acumulo de espécies de morcegos capturados em novembro de 2005, na Fazenda Marimbondo, Visconde de Mauá, município de Resende, RJ.

Figure 2. Randomized accumulation curve (with standard deviation) of bat species captured in November 2005 at Fazenda Marimbondo, Visconde de Mauá, Resende, RJ.

A Conservation International desenvolveu na última década um Programa de Avaliação Rápida (RAP), que consiste na execução de estudos de curta duração, com o objetivo de registrar do maior número de espécies em áreas pouco conhecidas (Alonso et al. 2001, Chermoff et al. 2001, Montambault \& Missa 2002). O objetivo desta abordagem não é gerar uma lista completa de espécies para os locais visitados, mas sim fornecer dados primordiais sobre a diversidade de uma área (Martins et al. 2006). Nesse sentido o presente estudo vem contribuir para o conhecimento da fauna de morcegos do município de Resende, no Estado do Rio de Janeiro.

\section{Agradecimentos}

Este trabalho foi financiado por Critical Ecosystem Partnership Fund (CEPF) e Aliança para Conservação da Mata Atlântica (Conservation International - Brazil e SOS Mata Atlântica). Agradecemos à equipe do Laboratório de Ecologia de Mamíferos (UERJ) que ajudou no trabalho de campo e ao Instituto Biomas pelo suporte. JLL agradece ao CNPq pela bolsa de doutorado. LMC agradece à FAPERJ pela bolsa de doutorado. CELE agradece ao CNPq a bolsa de produtividade em pesquisa e à FAPERJ a bolsa de Jovem Cientista do Nosso Estado. HGB agradece as bolsas de Produtividade CNPq, Prociência UERJ e Cientista do Nosso Estado FAPERJ.

\section{Referências Bibliográficas}

ALONSO, L.E., ALONSO, A., SCHULEMBERG, T.S. \& DALLMEIER, F. 2001. Biological and social assessments of the Cordillera de Vilcabamba, Peru. Rapid Assessment Program Bulletin of Biological Assessment, Washington, v.6, p.1-298.

BAUER, C. \& PACHECO, J.F. 2000. Lista das aves da região de Visconde de Mauá, Serra da Mantiqueira, no limite dos Estados do Rio de Janeiro e Minas Gerais. Atual. Ornitol. 97:7.
BERGALlO, H.G., FIDALGO, E.C.C., ROCHA, C.F.D., UZEDA, M.C., COSTA, M.B., ALVES, M.A.S., VAN SLUYS, M., SANTOS, M.A., COSTA, T.C.C. \& COZZOLINO, A.C.R. 2009. Estratégias e ações para a conservação da biodiversidade no Estado do Rio de Janeiro. Instituto Biomas, Rio de Janeiro.

BONACCORSO, F.J. 1979. Foraging and reproductive ecology in a Panamanian bat community. Bull. Fla. St. Mus. Biol. Sci. 24(4):359-408.

BONACCORSO, F.J. \& SMYTHE, N. 1972. Punch-Marking bats: An Alternative to Banding. J. Mammal. 53:389-390. http://dx.doi. org/10.2307/1379186

CHAO, A. 1984. Nonparametric estimation of the numbers of classes in a population.Scand. J. Stat. 11:265-270.

CHERMOFF, B., WILLINK, P.W. \& MONTAMBAULT, J.R. 2001. A biological assessment of the Rio Paraguay basin, Alto Paraguay, Paraguay. Rapid Assessment Program Bulletin of Biological Assessment, Washington. v.19, p.1-156.

DELCIELLOS, A.C., NOVAES, R.L.M., LOGUERCIO, M.F.C., GEISE, L., SANTORI, R.T., SOUZA, R.F., PAPI, B.S., RAÍCES, D.S.L., VIEIRA, N.R., FELIX, S., DETOGNE, N., SILVA, C.C.S., BERGALLO, H.G. \& ROCHA-BARBOSA, O. 2012. Mammals of Serra da Bocaina National Park, state of Rio de Janeiro, southeastern Brazil. Check List 8(4):675692.

DIAS, D. \& PERACCHI, A.L. 2008. Quirópteros da Reserva Biológica do Tinguá, Estado do Rio de Janeiro, sudeste do Brasil (Mammalia: Chiroptera). Rev. Brasil. Zool. 25:333-369. http://dx.doi.org/10.1590/ S0101-81752008000200023

DIAS, D., PEREIRA, S.N., MAAS, A.C.S., MARTINS, M.A., BOLZAN, D.P. \& PERACCHI, A.L. 2010. Quirópteros das regiões Centro-Sul e Médio Paraíba do Estado do Rio de Janeiro (Mammalia, Chiroptera). Chirop. Neotrop. 16(1):579-585.

ESBERÁRD, C.E.L. 2004. Morcegos no Estado do Rio de Janeiro. Tese de Doutorado, Universidade do Estado do Rio de Janeiro, Rio de Janeiro.

ESBÉRARD, C.E.L., CHAGAS, A.S., BAPTISTA, M. \& LUZ, E.M. 1996. Levantamento de Chiroptera na Reserva Biológica de Araras, Petrópolis, Rio de Janeiro - I - riqueza de espécies. Rev. Cient. C. Pes. 2:65-87.

ESBÉRARD, C.E.L., CHAGAS, A.S. \& LUZ, E.M. 1999. Uso de residências por morcegos no Estado do Rio de Janeiro (Mammalia: Chiroptera). Rev. Bras. Med. Vet. 21(1):17-20.

ESBÉRARD, C.E.L. \& BERGALLO, H.G. 2005. Research on bats in the state of Rio de Janeiro, Southeastern Brazil. Mastozool. Neotrop. 12(2):237243.

GEISE, L, PEREIRA, L.G., BOSSI, D.E. \& BERGALLO, H.G. 2004. Patterns of elevational distribution and richness of nonvolant mammals in Itatiaia National Park and surroundings, in Southeastern Brazil. Braz. J. Biol. 64:1-15. http://dx.doi.org/10.1590/S1519-69842004000400007

INSTITUTO CHICO MENDES DE CONSERVAÇÃO DA BIODIVERSIDADE - ICMBIO. 2012. APA da Serra da Mantiqueira. http://www.icmbio.gov.br/portal/biodiversidade/unidades-deconservacao/biomas-brasileiros/mata-atlantica/unidades-de-conservacaomata-atlantica/2177-apa-da-serra-da-mantiqueira.html (último acesso em 06/11/2012).

LUZ, J.L., COSTA, L.M., LOURENÇO, E.C., GOMES, L.A.C. \& ESBÉRARD, C.E.L. 2009. Bats from the Restinga of Praia das Neves, state of Espírito Santos, Southern Brazil. Check List 5(2):364-369.

LUZ, J.L., COSTA, L.M., LOURENÇO, E.C. \& ESBÉRARD, C.E.L. 2011. Morcegos (Mammalia, Chiroptera) da Reserva Rio das Pedras, Rio de Janeiro, Sudeste do Brasil. Biota Neotrop. 11(1): http://www. biotaneotropica.org.br/v11n1/pt/abstract?article+bn01711012011 (último acesso em 17/08/2012)

MARTINS, A.C.M., BERNARD, E. \& GREGORIN, R. 2006. Inventários biológicos rápidos de morcegos (Mammalia, Chiroptera) em três unidades de conservação do Amapá, Brasil. Rev. Bras. Zool. 23(4):1175-1184. http://dx.doi.org/10.1590/S0101-81752006000400026

MARTINS, M.A. 2011. Riqueza, diversidade de espécies e variação altitudinal de morcegos (Mammalia, Chiroptera) no Parque Nacional do Itatiaia, Rio de Janeiro, Brasil. Dissertação de Mestrado, Universidade Federal Rural do Rio de Janeiro, Rio de Janeiro.

McCAIN, C.M. 2005. Elevational gradients in diversity of small mammals. Ecology 86(2):366-372. http://dx.doi.org/10.1890/03-3147 
McCAIN, C.M. 2007. Could temperature and water availability drive elevational diversity? A global case study for bats. Global Ecol. Biogeogr. 16:1-13. http://dx.doi.org/10.1111/j.1466-8238.2006.00263.x

MODESTO, T.C., PESSÔA, F.S., ENRICI, M.C., ATTIAS, N., JORDÃONOGUEIRA, T., COSTA, L.M., ALBUQUERQUE, H.G. \& BERGALLO, H.G. 2008. Mamíferos do Parque Estadual do Desengano, Rio de Janeiro, Brasil. Biota Neotrop. 8(4): http://www.biotaneotropica.org.br/v8n4/pt/ abstract?article+bn01408042008 (último acesso em 17/08/2012)

MONTAMBAULT, J.R. \& MISSA, O. 2002. A Biodiversity Assessment of the Eastern Kanuku Mountains, Lower Kwitaro River, Guyana. Rapid Assessment Program Bulletin of Biological Assessment, Washington, v.26, p.1-88.

MORATELLI, R. \& PERACCHI, A.L. 2007. Morcegos (Mammalia, Chiroptera) do Parque Nacional da Serra dos Órgãos. In Ciência e conservação na Serra dos Órgãos (C. Cronemberger \& E.B. Viveiros De Castro, eds.). Instituto Chico Mendes de Conservação da Biodiversidade, Brasília, p.193-210.

NOBRE, P.H., RODRIGUES, A.S., COSTA, A.I., MOREIRA, A.E.S., MOREIRA, H.H. 2009. Similaridade da fauna de Chiroptera (Mammalia), da Serra Negra, municípios de Rio Preto e Santa Bárbara do Monte Verde, Minas Gerais, com outras localidades da Mata Atlântica. Biota Neotrop. 9(3): http://www.biotaneotropica.org.br/v9n3/pt/ abstract?article+bn03309032009 (último acesso em 17/08/2012)

OLIFIERS, N., CUNHA, A.A., GRELLE, C.E., BONVICINO, C.R., GEISE, L., PEREIRA, L.G., VIEIRA, M.V., D'ANDREA, P.S. \& CERQUEIRA, R. 2007. Lista de espécies de pequenos mamíferos não-voadores do Parque Nacional da Serra dos Órgãos. In Ciência e conservação na Serra dos Órgãos (C. Cronemberger \& E.B. Viveiros De Castro, eds.). Instituto Chico Mendes de Conservação da Biodiversidade, Brasília, p.183-192.
PAGLIA, A.P., FONSECA, G.A.B., RYLANDS, A.B., HERRMANN, G., AGUIAR, L.M.S., CHIARELLO, A.G., LEITE, Y.L.R., COSTA, L.P., SICILIANO, S., KIERULFF, M.C.M., MENDES, S.L., TAVARES, V.C., MITTERMEIER, R.A. \& PATTON, J.L. 2012. Lista Anotada dos Mamíferos do Brasil. 2. ed. Occas. Pap. Conserv. Biol. 6:1-76.

PATTERSON, B.D., STOTZ, D.F., SOLARI, S., FITZPATRICK, J.W. \& PACHECO, V. 1998. Contrasting patterns of elevational zonation for birds and mammals in the Andes of southeastern Peru. J. Biogeogr. 25:593-607. http://dx.doi.org/10.1046/j.1365-2699.1998.2530593.x

SIMMONS N.B. 2005. Order Chiroptera. In Mammal species of the world: a taxonomic and geographic reference (D.E. Wilson \& D.M. Reeder, eds.). Johns Hopkins University Press, Baltimore, p.312-529.

SIMMONS, N.B. \& VOSS, R.S. 1998. The mammals of Paracou, French Guiana: a Neotropical lowland rainforest fauna, Part 1. Bats. B. Am. Mus. Nat. Hist. 237:1-219.

STRAUBE, F.C. \& BIANCONI, G.V. 2002. Sobre a grandeza e a unidade utilizada para estimar esforço de captura com utilização de redes-deneblina. Chiropt. Neotrop. 8(1-2):150-152.

URURAHY, J.C.C., COLLARES, J.E.R., SANTOS, M.M. \& BARRETO, R.A.A. 1983. Folhas SF.23/24 Rio de Janeiro/Vitória, geologia, geomorfologia, pedologia, vegetação e uso potencial da terra. In Projeto RADAMBRASIL, Vegetação: as regiões fitoecológicas, sua natureza e seus recursos econômicos. Instituto Brasileiro de Geografia e Estatística, Rio de Janeiro.

VOSS, R.S. \& EMMONS, L.H. 1996. Mammalian diversity in neotropical lowland rainforests: a preliminary assessment. B. Am. Mus. Nat. Hist. 230:1-115.

VRCIBRADIC, D.,ARIANI, C.V., VAN SLUYS, M. \& ROCHA. C.F.D. 2010. Amphibia, Leptodactylidae, Paratelmatobius mantiqueira: Distribution extension. Check List 6(1):1-2. 


\section{Apêndice}

Apêndice 1. Espécimes depositados na Coleção de Referência do Laboratório de Diversidade de Morcegos (LDM), Instituto de Biologia, Universidade Federal Rural do Rio de Janeiro: Desmodus rotundus (LDM4136); Eptesicus furinalis (LDM4149); Myotis riparius (LDM4147); Molossus molossus (LDM4160); Platyrrhinus recifinus (LDM4141); Sturnira lilium (LDM4140); Vampyressa pusilla (LDM4144).

Appendix 1. Vouchers deposited in the collection of Laboratório de Diversidade de Morcegos at Universidade Federal Rural do Rio de Janeiro: Desmodus rotundus (LDM4136); Eptesicus furinalis (LDM4149); Myotis riparius (LDM4147); Molossus molossus (LDM4160); Platyrrhinus recifinus (LDM4141); Sturnira lilium (LDM4140); Vampyressa pusilla (LDM4144). 